\title{
High Frequency Fed Unity Power Factor AC-DC Converter for Wireless Power Transfer Applications
}

\author{
Rose Maria Alex ${ }^{1}$, Ginu Ann George ${ }^{2}$ \\ PG Scholar, Dept of Electrical and Electronics Engg, St. Joseph's College of Engineering and Technology, \\ Palai, India ${ }^{1}$
}

Assistant Professor, Dept of Electrical and Electronics Engg, St. Joseph's College of Engineering and Technology, Palai, India ${ }^{2}$

\begin{abstract}
Wireless power transfer over near to midrange distances have been receiving much attention owing to their potential applications such as biomedical implants, charging pad platforms for mobile devices and electric vehicle recharging. The newest technologies mostly depend on inductive coupling techniques to transmit power between transmitting and receiving coils. Wireless power transfer (WPT) receivers are devices that can transmit power to electrical devices without any wired connections. But there is a lack of research targeting the optimal design of the power converter at the receiver side. The novel scheme presents a power converter and its control circuit for high frequency fed AC to DC conversion for wireless power transfer. Wireless power transfer is achieved via resonant inductive coupling between the transmitting and receiving coils. Based on the resonant technique, the input current is shaped to be sinusoidal and is forced to follow the high-frequency sinusoidal input voltage so as to achieve unity power factor. A control scheme is also proposed for the converter which can be realized by simple operational amplifiers and digital logic gates. The distinctive features of this converter are favourable for future high-frequency AC power transfer system operating in the range from a few hundred $\mathrm{kHz}$ to the $\mathrm{MHz}$ range.
\end{abstract}

Keywords: Wireless Power Transfer (WPT), magnetic resonant coupling, Power Factor (PF) correction, High frequency converter.

\section{INTRODUCTION}

Wireless power transmission (WPT) is the transmission of electric power through vacuum or atmosphere without the use of wire. The various techniques used in WPT are inductive coupling for short range, resonant induction for mid-range and electromagnetic wave power transfer. Magnetic field coupling has the higher efficiency which works on the principle of electromagnetism. Power transfer range can be increased wit resonance phenomenon applied on magnetic coupling.

Inventor Nikola Tesla was the first to experiment on wireless power transmission at the beginning of 20th century [2]. He used spark-excited radio frequency resonant transformers, now called Tesla coils, which generated high $\mathrm{AC}$ voltages. With these he was able to transmit power for short distances without wires. At his Colorado Springs laboratory during $1899-1900$, by using voltages of the order of 10 megavolts generated by an enormous coil, he was able to light three incandescent lamps at a distance of about one hundred feet. The resonant inductive coupling which Tesla pioneered is used throughout electronics. Its use in wireless power has been recently rediscovered and it is currently being widely applied to short-range wireless power systems.

An equivalent-circuit model can be developed for a wireless power transfer system by coupled magnetic resonances. The input to the system is from an $\mathrm{AC}$ power source and is allowed to be transferred without wire by resonant inductive coupling. The inductor is coupled with a capacitor to be tuned to a frequency. The frequencies can be matched by both the transmitting and receiving coils. After the receiving coil obtains the AC, it will be converted back to DC using a full wave rectifier and regulator circuit.

Thus the power converter at the receiver side is a diode rectifier circuit with an output storage capacitor as shown in Fig. 1. This capacitor is charged to a value close to the peak of the ac input voltage resulting in a pulsating input current of large magnitude at the peak of the ac input voltage. Hence at the receiver side, draws highly distorted current from the ac power source resulting in a poor input power factor (PF). The energy efficiency and power transfer capability of a poor PF system are relatively low because of the high conduction loss in the power converters and transmission wires.

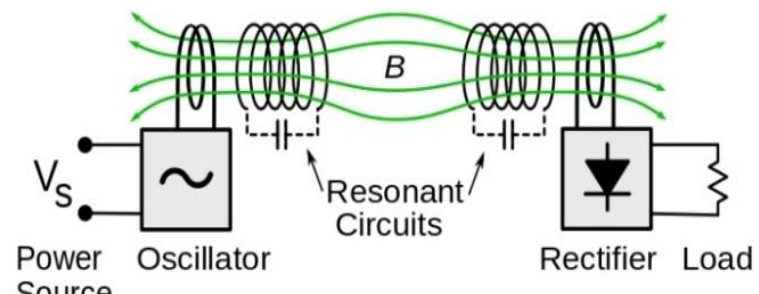

Fig. 1: Wireless power transfer system 
The rest of the paper is organized as follows: Section II The entire process can be summarised as, in the first stage, describes the concept of wireless power transfer system by ie., in the PFC stage, capacitors $\mathrm{C}_{\mathrm{r} 1}$ and $\mathrm{C}_{\mathrm{r} 2}$ are resonant inductive coupling. The operating principle and alternatively charged by input AC voltage source by various modes of operation of the proposed high frequency fed AC-DC power converter will be explicitly described in Section III. Section IV discusses the simulation results, and the conclusion is arrived at Section V.

\section{RESONANT INDUCTIVE COUPLING}

Inductive or magnetic coupling is based on the principle of electromagnetism. When a conductor is proximity to a magnetic field, it induces a magnetic field in that wire. Transferring energy between wires through magnetic fields is inductive coupling. Magnetic resonant coupling uses the same principles as inductive coupling, but it uses resonance to increase the range at which the energy transfer can efficiently take place[4], [5].

Resonant inductive coupling is the near field wireless transmission of electrical energy between two coils that are tuned to resonate at the same frequency and is depicted in Fig. 1. Resonant transfer works by making a coil ring with an oscillating current. This generates an oscillating magnetic field. Because the coil is highly resonant, any energy placed in the coil dies away relatively slowly over very many cycles; but if a second coil is brought near it, the coil can pick up most of the energy before it is lost, even if it is some distance away. The oscillations in the magnetic field will die away at a rate determined by the gain-bandwidth ( $\mathrm{Q}$ factor) due to resistive and radiative losses. Because the $\mathrm{Q}$ can be very high, even when low power is fed into the transmitter coil, a relatively intense field builds up over multiple cycles, which increases the power that can be received. Thus, at resonance far more power can be transferred wireless.

Resonant circuit can be either series or parallel. The difference is how they are connected to the power amplifier of the system. A parallel resonator is used in the parallel case, and in the series case a series resonator is used. The two types of circuits behave similarly but some differences exist, ie., to drive a parallel circuit the driving circuit should have a high output resistance, and therefore behave like a current source rather than a voltage source to minimize the reduction of the resonator quality factor. For the series circuit the opposite is true, the driving circuit should have a low output resistance. In addition to transferring the power efficiently, the transferred voltage should not vary too much. Keeping the voltage stable even with variations in the coupling coefficient is desirable to reduce the power loss in the voltage regulator, as well as preventing the load circuit from being damaged from high voltage.

\section{III.RECIEVER TOPOLOGY}

The proposed two stage topology is shown in Fig. 2[1]. The two stages are PFC (Power Factor Correction) and regulation stage respectively. The PFC stage consists of two switches, four diodes, two resonant inductors and two resonant capacitors. The regulation stage comprises of two switches, three diodes, one capacitor and one inductor. alternative switching of switches $S_{1}$ and $S_{2}$ respectively. In the second stage ie., in the regulation stage the previously charged capacitors commutates alternately as energy sources for second stage. Buck-Boost converter is used for regulation stage and in total there are four modes of operation for the proposed topology.

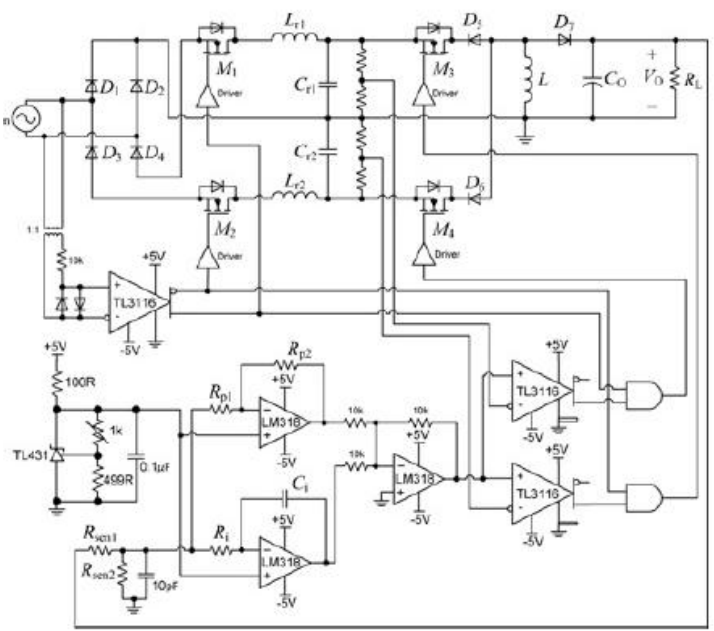

Fig. 2: Schematic of high frequency fed AC-DC power converter

\section{A. Modes of Operation}

As previously stated there are four modes of operation. The switches $S_{1}$ and $S_{2}$ are used to select the resonant tanks $\mathrm{L}_{\mathrm{r} 1} \mathrm{C}_{\mathrm{r} 1}$ and $\mathrm{L}_{\mathrm{r} 2} \mathrm{C}_{\mathrm{r} 2}$ respectively during the PFC stage. Meanwhile the switches $S_{3}$ and $S_{4}$ are used to control the Buck-Boost converter in regulation switch during the positive and negative half cycles respectively.

MODE 1: We assume capacitors $C_{r 1}$ and $C_{r 2}$ are initially charged to $\mathrm{V}_{\mathrm{cr} \text {,min }}$ and $\mathrm{V}_{\mathrm{cr} \text {,max }}$ respectively. In the first stage, ie., $\mathrm{PF}$ correction stage, $\mathrm{S}_{1}$ is turned $\mathrm{ON}$ and $\mathrm{S}_{2}$ remains OFF. $D_{1}$ and $D_{4}$ are forward biased and diodes $D_{2}$ and $D_{3}$ are reverse biased. $\mathrm{L}_{\mathrm{r} 1}$ and $\mathrm{C}_{\mathrm{r} 1}$ together forms a series resonant circuit. The inductor current starts from an initial value, say zero, follows the sinusoidal waveform and decreases to zero as $D_{1}$ and $D_{4}$ are reverse biased. The voltage across the capacitor $\mathrm{C}_{\mathrm{r} 1}$ is charged from initial value $\mathrm{V}_{\mathrm{cr} \text {,min }}$ to a certain level. Mode 1 is illustrated in Fig. 3.

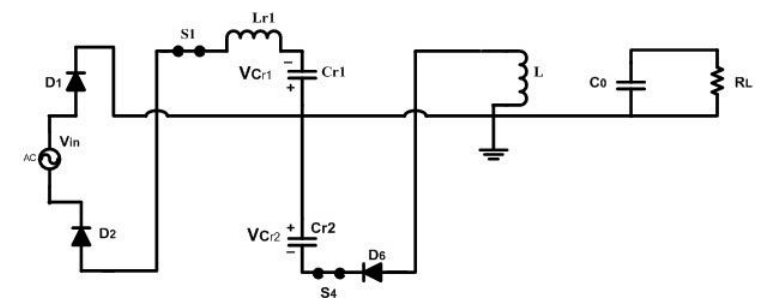

Fig. 3: Mode 1

In the second stage, ie., regulation stage, $\mathrm{S}_{4}$ is turned $\mathrm{ON}$ while $S_{3}$ in OFF state. Diode $D_{6}$ is forward biased while diodes $D_{5}$ and $D_{7}$ reverse biased. $C_{r 2}$ is discharged in one direction because of polarity of diode $\mathrm{D}_{6}$. Energy from the 
PFC stage get transferred to the regulation stage and is stored in the inductor L. The energy to the output load resistor $R_{L}$ is delivered by output capacitor $C_{O}$.

MODE 2: The PFC stage does not get altered in the Mode 2 operation and is shown in Fig. 4. Switches $S_{1}, S_{2}$ and diodes $D_{1}, D_{2}, D_{3}, D_{4}$ have similar functions as that of Mode 1. Capacitor $\mathrm{C}_{\mathrm{r} 1}$ charges to $\mathrm{V}_{\mathrm{cr} \text {,max }}$ as positive half cycle ends. Switch $S_{1}$ gets commutated naturally.

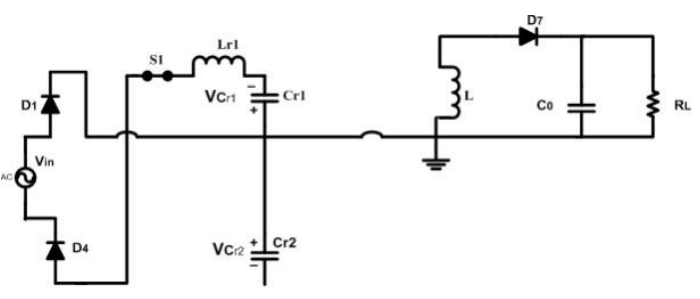

Fig. 4: Mode 2

\section{B. Control Methodology}

A control methodology based on analog circuits is implemented. A phase detector circuit is used in the PFC stage to control the ON/OFF time of switches $S_{1}$ and $S_{2}$. The AC source voltage $V_{\text {in }}$ is sensed and given to the phase detector circuit where the output of phase detector circuit is connected to the driver circuit of switches $S_{1}$ and $\mathrm{S}_{2}$.

The control signals for the switches $S_{3}$ and $S_{4}$ are derived from the pulse width modulation generators in which output from the phase detector circuit are applied. ie., in the regulation stage, the instantaneous voltage of resonant capacitors are sensed and compared with $\mathrm{V}_{\text {cr,min }}$ to generate the pulses of switches $S_{3}$ and $S_{4}$.

\section{SIMULATION RESULTS}

Diodes $D_{1}$ and $D_{4}$ gets reverse biased, while $S_{3}$ and $D_{5}$ in the regulation stage remains OFF. The current of inductor $L$ results in the forward conduction of diode $D_{7}$. And in this stage energy stored in the inductor is delivered to the output capacitor $\mathrm{C}_{\mathrm{O}}$ and load resistor $\mathrm{R}_{\mathrm{L}}$.

MODE 3: In the PFC stage, ie., in negative half cycle, switch $\mathrm{S}_{2}$ is turned $\mathrm{ON}$ and switch $\mathrm{S}_{1}$ is turned OFF. Diodes $D_{2}$ and $D_{3}$ are forward biased and diodes $D_{1}$ and $\mathrm{D}_{4}$ are reverse biased. Capacitor $\mathrm{C}_{\mathrm{r} 2}$ charges from $\mathrm{V}_{\mathrm{cr} \text {,min }}$ to $\mathrm{V}_{\text {cr,max. }}$ Mode 3 is shown in Fig. 5.

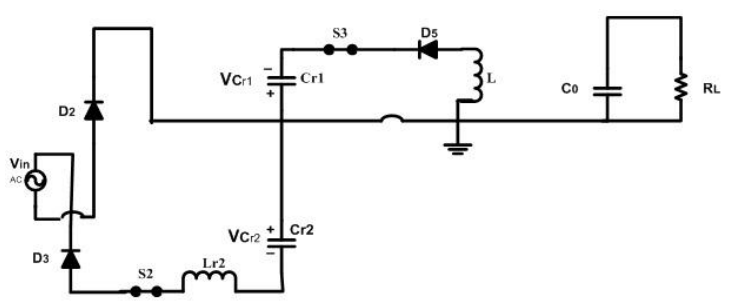

Fig. 5: Mode 3

Whereas in regulation stage, switch $\mathrm{S}_{3}$ turns $\mathrm{ON}$ and switch $S_{4}$ and diode $D_{6}$ remains in OFF state. Diode $D_{5}$ forward biased and diode $\mathrm{D}_{7}$ reverse biased. $\mathrm{C}_{\mathrm{r} 1}$ discharges to inductor $\mathrm{L}$ until the voltage of capacitor $\mathrm{C}_{\mathrm{r} 1}$ is equal to $\mathrm{V}_{\text {cr,min. }}$. At the same time load resistor $\mathrm{R}_{\mathrm{L}}$ is supplied by the output capacitor $\mathrm{C}_{\mathrm{O}}$.

MODE 4: Mode 4 is illustrated in Fig. 6. In the PFC stage, switches $S_{1}$ and $S_{2}$ and diodes $D_{1}$ and $D_{2}$ have same switching states as that of previous mode. Energy stored in inductor $\mathrm{L}$ during previous mode is discharged to $\mathrm{C}_{\mathrm{O}}$ and $\mathrm{R}_{\mathrm{L}}$ through diode $\mathrm{D}_{7}$. Switch $\mathrm{S}_{2}$ is commutated at the end of negative half cycle and later the entire modes are repeated.



Fig. 6: Mode 4
The proposed high frequency fed AC-DC power converter and its control circuit is implemented using OrCAD. Fig. 7 shows the pspice model of the proposed converter. The converter is designed such as to convert a $400 \mathrm{kHz}$ AC voltage source into a DC source. The input voltage provided to the converter is $50 \mathrm{~V}_{\mathrm{rms}}$.

The output power obtained is $30 \mathrm{~W}$. The output voltage obtained is $54 \mathrm{~V}$ and is shown in Fig. 8. The input-output voltage waveforms are captured and are shown in Fig. 9. Also the voltage across resonant capacitors is captured and is shown as Fig. 10. The power factor correction, ie., input current and voltage is depicted in Fig. 11. The simulation waveforms are in good agreement with the theoretical analysis.

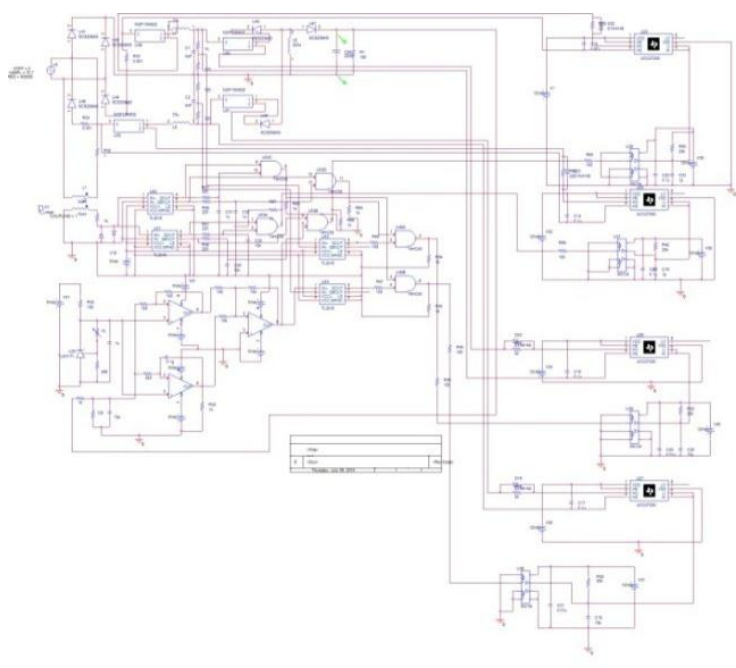

Fig. 7: Pspice model

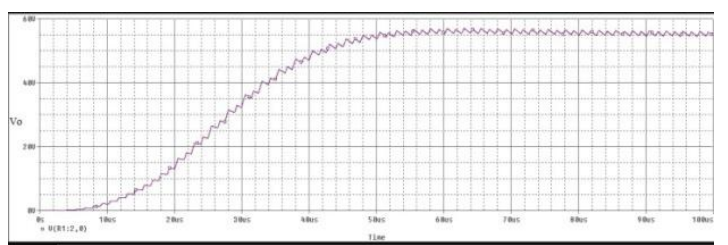

Fig. 8 : Output voltage 


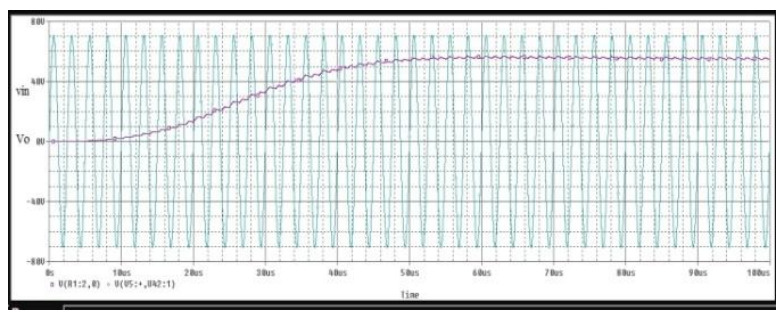

Fig. 9: Input output voltage

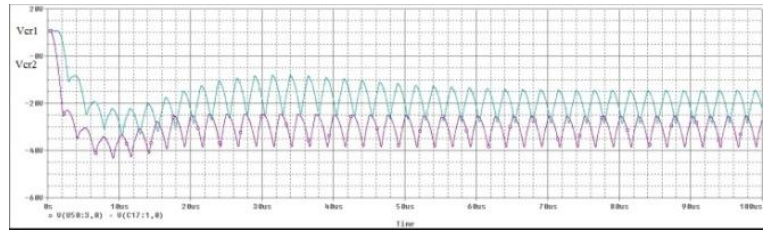

Fig. 10: Voltage across resonant capacitors

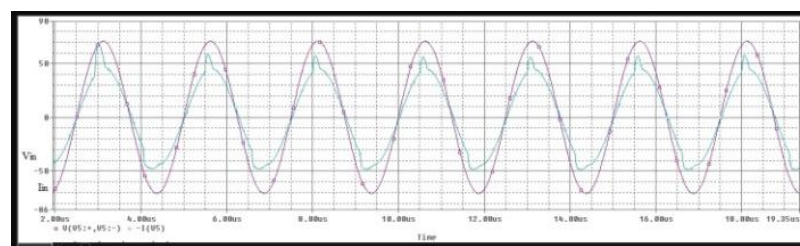

Fig. 11: Input voltage and current

\section{TABLE I CIRCUIT SPECIFICATIONS}

\begin{tabular}{|l|l|}
\hline Input Voltage & $70.7 \mathrm{~V}$ \\
\hline Source Frquency & $400 \mathrm{kHz}$ \\
\hline $\begin{array}{l}\text { Switching } \\
\text { Frequency }\end{array}$ & $400 \mathrm{kHz}$ \\
\hline Output Power & $30 \mathrm{~W}$ \\
\hline Output Voltage & $54 \mathrm{~V}$ \\
\hline
\end{tabular}

The circuit specifications are summarised in Table I. And the block diagram of entire wireless power transfer system is depicted in Fig. 12. AC is converted to High frequency AC with the help of a controller and is fed to the transmitter. The receiver is in vicinity of transmitter magnetic field and is rectified to feed the load.

The component specification used for simulation in OrCAD is summarised in Table II. The switching pulses to switches $S_{1}$ and $S_{2}$ are shown in Fig. 13 whereas pulses to switches $S_{3}$ and $S_{4}$ are depicted in Fig. 14.

\section{TABLE II COMPONENT SPECIFICATIONS}

\begin{tabular}{|l|l|}
\hline Transistors & NDF10NZ \\
\hline Diodes & SiC Diode \\
\hline Resonant Inductors & $33 \mathrm{uH}$ \\
\hline $\begin{array}{l}\text { Resonant } \\
\text { Capacitors }\end{array}$ & $5 \mathrm{nF}$ \\
\hline Driver IC & 6N134 \\
\hline Opt coupler IC & UCC20277 \\
\hline
\end{tabular}

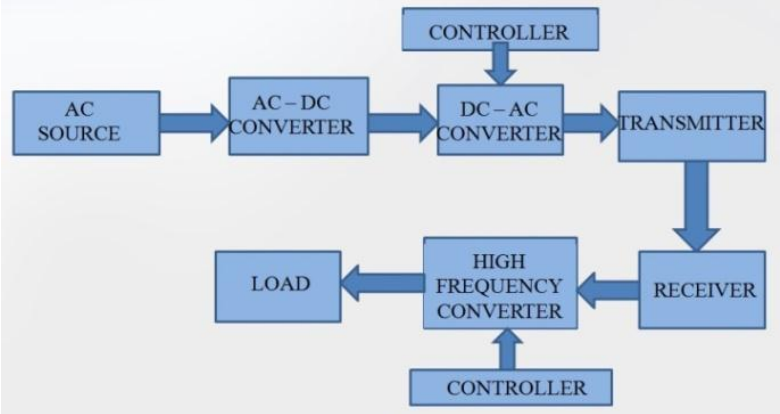

Fig. 12: Block diagram of hardware development

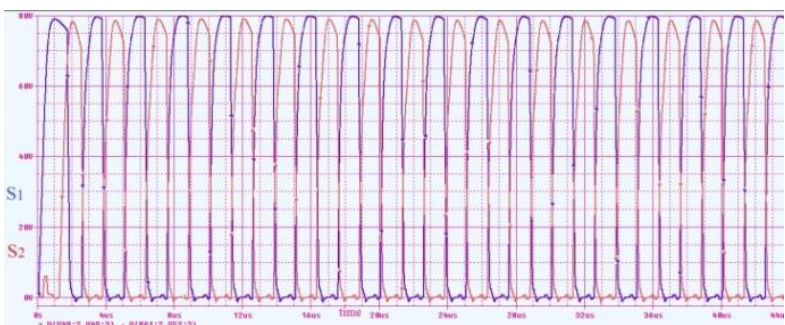

Fig. 13: Switching pulses to $S_{1}$ and $S_{2}$

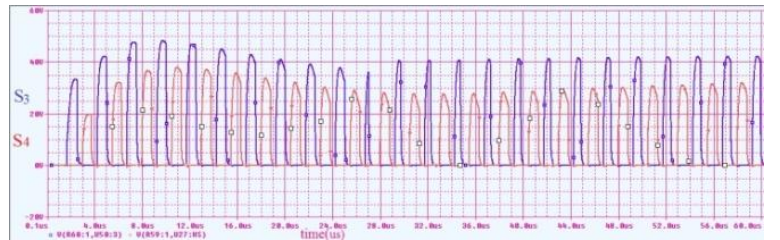

Fig. 14: Switching pulses to $S_{3}$ and $S_{4}$

\section{CONCLUSION}

In this paper a wireless power transfer system using resonant inductive coupling is demonstrated. Unlike a traditional WPT system which uses a rectifier circuit in the receiver side, the proposed system uses a high frequency converter with two stages. The stages being the power factor correction stage and the regulation stage. The converter is able to acheive a high power factor and low input current distortion. The proposed converter along with its control circuit is simulated in OrCAD and the corresponding results were verified.

\section{REFERENCES}

[1] Chi-Kwan Lee, Sitthisak Kiratipongvoot and Siew-Chong Ton, "High frequency fed unity power factor AC-DC converter with one switching per cycle," IEEE Trans.Power Electron., Vol. 30, No. 4, April 2015.

[2] N. Tesla, Apparatus for transmitting electrical energy, U.S. Patent 1 119732, Dec. 1, 1914..

[3] J. C. Schuder, J. H. Gold and H. E. Stephenson," An inductively coupled RF system for transmission of $1 \mathrm{~kW}$ of power through skin,” IEEE Trans. Biomed. Eng. Vol. BME-18, No.4, pp. 265-273, Jul. 1971.

[4] S.Cheon, Y. H. Kim, S. Y. Kang, M. L. Lee, J. M. Lee and T. Zyung ,Circuit model based analysis of a wireless energy transfer system via coupled magnetic resonance," IEEE Trans.Ind.Electron., Vol. 58 No. 7, pp. 2906-2914, Jul. 2011.

[5] Y. H. Kim, S. Y. Kang, S.Cheon, M. L. Lee, J. M. Lee and T. Zyung ,'Optimization of wireless power transfer through resonant coupling," in Proc. Compact. Power. Electron. 2009, pp. 426-431. 